\title{
Extending cycles locally to Hamilton cycles
}

\author{
Matthias Hamann Florian Lehner* \\ Department of Mathematics \\ University of Hamburg, Germany \\ \{matthias.hamann, florian.lehner\}@math.uni-hamburg.de \\ Julian Pott \\ julianpott@gmail.com
}

Submitted: Dec 12, 2013; Accepted: Mar 1, 2016; Published: Mar 18, 2016

Mathematics Subject Classifications: 05C63, 05C45

\begin{abstract}
A Hamilton circle in an infinite graph is a homeomorphic copy of the unit circle $S^{1}$ that contains all vertices and all ends precisely once. We prove that every connected, locally connected, locally finite, claw-free graph has such a Hamilton circle, extending a result of Oberly and Sumner to infinite graphs. Furthermore, we show that such graphs are Hamilton-connected if and only if they are 3-connected, extending a result of Asratian. Hamilton-connected means that between any two vertices there is a Hamilton arc, a homeomorphic copy of the unit interval $[0,1]$ that contains all vertices and all ends precisely once.
\end{abstract}

Keywords: infinite graphs; Hamilton cycles; Hamilton connected

\section{Introduction}

The proofs of many classical sufficient conditions for the existence of a Hamilton cycle can be outlined as follows. Start with an arbitrary cycle, extend the cycle by some additional vertices and iterate this extension procedure until the cycle covers all vertices. It is often the case that the extension happens locally, that is, most of the original cyclein fact everything outside a bounded distance from some newly added vertex-remains unchanged.

While such a strategy will obviously give a Hamilton cycle for finite graphs, the situation is more complicated with infinite graphs, particularly because it is not entirely clear what an infinite analogue of a Hamilton cycle should be.

*The author acknowledges the support of the Austrian Science Fund (FWF), project W1230-N13. 
Considering spanning rays or spanning double rays as infinite analogues of Hamiltonian cycles has yielded some results (e. g., see Thomassen [24]) but it has the obvious drawback that a graph with more than two ends can never be Hamiltonian. However, there is a different approach suggested by Diestel and Kühn $[11,12]$. They define a circle in an infinite graph $G$ to be a homeomorphic image of the unit circle in the end compactification of $G$. This approach has not only been successful in generalizing Hamiltonicity results to locally finite graphs, it has also yielded generalizations of many theorems about the cycle space (see [9] for an overview).

Unfortunately, the extension strategies mentioned above do not immediately give a Hamiltonian circle in this sense. There will be a limit object if the extension procedure only alters the cycle locally, but it is not guaranteed that this limit object will be a circle. In particular, ensuring injectivity at the ends of $G$ can be challenging. So far, there are some results on Hamilton circles in infinite graphs, see [3, 5, 7, 15, 19]

In this paper we present a strategy that ensures that the limit will be a Hamilton circle. We call it a $k$-local skip-and-glue strategy and it roughly states that every finite 2-regular subgraph can be extended to a larger 2-regular graph with an extension of bounded size, or to a 2-regular graph with fewer components. In Section 3 we define this $k$-local skipand-glue strategy and prove that a locally finite graph with such a strategy contains a Hamilton circle.

We then proceed to prove that locally finite claw-free graphs always admit such a strategy. So we shall show that the following two theorems can be extended to locally finite graphs and affirmatively answer questions of Stein [23, Question 5.1.3] and Bruhn, see Stein [23, Question 5.1.4].

Theorem 1. [22, Theorem 1] Every finite connected locally connected claw free graph on at least three vertices is Hamiltonian.

Theorem 2. [1, Theorem 3.4] A finite locally connected claw-free graph on at least four vertices is Hamilton-connected if and only if it is 3-connected.

We also give some corollaries of the two theorems whose infinite but locally finite counterparts are corollaries to the infinite versions of those theorems. Similar questions on Hamilton circles in infinite graphs were investigated by Heuer $[17,18]$.

\section{The topological space $|G|$}

Let $G=(V, E)$ be a locally finite graph. A ray is a one-way infinite path. Two rays are equivalent if they lie eventually in the same component of $G-S$ for every finite vertex set $S \subseteq V$. This is an equivalence relation whose equivalence classes are the ends of $G$. For $S \subseteq V$ and an end $\omega$, let $C(S, \omega)$ be the component of $G-S$ that contains some ray, and hence a tail of every ray, in $\omega$ and let $\Omega(S, \omega)$ be the set of ends with at least one ray in $C(S, \omega)$.

The space $|G|$ is a topological space on $G$ with its ends such that it coincides on $G$ with its 1-complex and such that the sets

$$
C(S, \omega) \cup \Omega(S, \omega) \cup E^{\prime}(S, \omega)
$$


for all finite $S \subseteq V$ and ends $\omega$ form a basis for the open neighbourhoods around each end $\omega$, where $E^{\prime}(S, \omega)$ is any union of half edges $(z, y]$, one for every edge $x y$ with $x \in S$ and $y \in C(S, \omega)$, with $z$ an inner point of $x y$. It can be proved (see [10]) that $|G|$ is the Freudenthal compactification [14] of the 1-complex $G$.

A circle in $|G|$ is a homeomorphic image of $S^{1}$ and an arc in $|G|$ is a homeomorphic image of $[0,1]$. A circle that contains every vertex and every end of $G$ is a Hamilton circle and an arc whose endpoints are vertices and that contains every vertex and every end of $G$ is a Hamilton arc. We call $G$ Hamilton-connected if there is a Hamilton arc between every two vertices of $G$.

A standard subspace of $|G|$ is a subspace of the form $\bar{U} \cup \stackrel{\circ}{F}$, where $(U, F)$ is a subgraph of $G$. We will need the following two lemmas on standard subspaces:

Lemma 3. A standard subspace of $|G|$ is topologically connected if and only if one of the following statements holds.

(i) It contains an edge from every finite cut of $G$ which meets both sides [8, Lemma 8.5.5].

(ii) It is arc-connected [13, Theorem 2.6].

The degree of an end $\omega$ in a standard subspace $X \subseteq|G|$ is the supremum of the cardinalities of sets of vertex disjoint arcs ending in $\omega$. This notion of degree for the whole space $|G|$ coincides with the notion of vertex-degree of ends in $G$.

Combining [11, Theorem 7.1] and [2, Theorem 5], we obtain the following theorem (see [9, Theorem 2.5]):

Theorem 4. Let $G$ be a locally finite graph and $F \subseteq E(G)$. Then the following statements are equivalent:

(i) Every vertex and every end has even degree in $\bar{F}$.

(ii) Every finite cut meets $F$ in an even number of edges.

The following characterization of subspaces that are circles comes in extremly handy.

Lemma 5. [4, Proposition 3] A standard subspace $X$ of $|G|$ is a circle if and only if it is topologically connected and every vertex and end of $G$ in $X$ has degree 2.

Let $H$ be a subgraph of $G$. A vertex $v \in G$ has depth $d(v, G-H)$ in $H$, that is the distance from $v$ to anything outside of $H$, and the depth of an edge is the maximum depth of its incident vertices.

The following proposition shows a possibility how to obtain a Hamilton circle as a limit of finite cycles.

Proposition 6. Let $G$ be a locally finite graph and let $\left(C_{i}\right)_{i \in \mathbb{N}}$ be a sequence of finite cycles such that

(i) $V\left(C_{i+1}\right)$ contains $V\left(C_{i}\right)$, 
(ii) an edge with depth at least $2 k$ in $C_{i}$ is contained in $C_{i+1}$ if and only if it is contained in $C_{i}$,

(iii) every vertex of $G$ is contained in some $C_{i}$,

(iv) for every end $\omega$ of $G$ and every finite set $V^{\prime} \subseteq V$ there is a finite cut $F$ separating $\omega$ from $V^{\prime}$ and an index $i_{0}$ such that $\left|E\left(C_{i_{0}}\right) \cap F\right|=2$ and $E\left(C_{i_{0}}\right) \cap F=E\left(C_{i}\right) \cap F$ for every $i>i_{0}$.

Let $C$ be the limit of the sequence $C_{i}$, that is, $C$ is the set of all those edges that are contained in all but finitely many $C_{i}$. Then $\bar{C}$ is a Hamilton circle.

Proof. Together, (i) and (iii) imply $\bigcup V\left(C_{i}\right)=V(G)$. By (iii) and as $G$ is locally finite there is for every vertex $v$ an index $j$ such that $C_{j}$ contains all vertices with distance $k+1$ or less from $v$. By (ii) and local finiteness every vertex has degree 2 in $C$ and $V(C)=V(G)$. Let $F$ be a finite cut with bipartition $(A, B)$. Then there is some $j \in \mathbb{N}$ such that all vertices incident with edges in $F$ have depth at least $2 k$ in $C_{j}$. As $C_{j+1}$ is a cycle and meets both $A$ and $B$, it must contain an even number of edges from $F$. These edges are contained in $C$ by (ii). As $C$ meets every finite cut in a positive even number of edges, every vertex and every end of $\bar{C}$ has even degree by Theorem 4 and $\bar{C}$ is topologically connected by Lemma 3 (i). Additionally, Lemma 3 (ii) implies that the standard subspace $\bar{C}$ is arc-connected and hence the degree of each vertex and end is at least 2 . We already saw that every vertex has degree 2 . Since every vertex lies in $\bar{C}$, so does every end. By (iv) we find for every end $\omega$ a sequence of cuts $\left(F_{i}\right)_{i \in \mathbb{N}}$ such that the components of $G-F_{i}$ whose closure contains $\omega$ converge to $\omega$ and such that each of these cuts contains precisely two edges of $C$. Thus, the degree of $\omega$ is at most 2 and hence precisely 2 . This implies that the standard subspace $\bar{C}$ is a circle by Lemma 5 . It is a Hamilton circle, as it contains every vertex and every end.

\section{Skip-and-glue extensions}

Let $F$ be a 2-regular finite sugraph of a graph $G$. Let $P$ be a path whose end vertices $v$ and $w$ are adjacent in $F$ but that is otherwise disjoint from $F$. Then the 2-regular subgraph $(F \cup P)-v w$ is the glue extension of $F$ by $P$ over the edge $v w$. A path $R \subseteq F$ is skippable if its two end vertices $x$ and $y$ are adjacent in $G$ but not in $F$. The edge $x y$ is a bypass of $R$. A 2-regular graph $F^{\prime}$ obtained from $F$ by successively replacing skippable paths by their bypasses is called a reduction.

A glue extension of a reduction $F^{\prime}$ of $F$ by $P$ that covers $F$ is a skip-and-glue extension of $F$ by $P$. Its depth is the maximum depth of any vertex of $F \cap P$ in its component of $F$. It is a skip-and-glue extension via $u$ if there is a vertex $u$ in $P \backslash F$. A skip-and-glue extension of $F$ via $u$ is $k$-local if its length, the length of $P$, is at most $k$ and $u$ is adjacent to $F$.

For a finite 2-regular subgraph $F \subseteq G$ with at least two components, a glue fusion of $F$ is a 2-regular subgraph $F^{\prime}$ such that $F^{\prime}$ coincides with $F-e-f$ on $V\left(F^{\prime}\right)$ where 
$e, f$ are edges from distinct components of $F$ and such that the edges in $F^{\prime} \backslash F$ form two disjoint paths connecting the two end vertices of $e$ with those of $f$. In particular, the glue fusion $F^{\prime}$ has less components than $F$ : precisely two components of $F$ are fused to one of $F^{\prime}$. We call $D$ a skip-and-glue fusion of $F$ if it is a glue fusion of a reduction $F^{\prime}$ of $F$ that covers $F$. This skip-and-glue fusion is $k$-local if the length of each of the two non-trivial paths in $D \backslash F^{\prime}$ is at most $k$. A $k$-local skip-and-glue fusion is centred around a vertex $u \in V(F)$ if each vertex of the two non-trivial paths in $F^{\prime} \backslash F$ has distance at most $k$ to $u$ and if $u$ lies in one of the two fused components.

A $k$-local skip-and-glue strategy (with respect to $S$ ) is a function which assigns

- to every pair $(F, u)$ of a finite 2-regular subgraph $F \subseteq G$ (covering $S$ ) all whose components contain at least $k$ vertices and a vertex $u \in N(F)$ a $k$-local skip-andglue extension of $F$ via $u$ and

- to every pair $(F, u)$ of a finite 2-regular disconnected subgraph $F \subseteq G$ (covering $S$ ) all whose components contain at least $k$ vertices and vertex $u \in V(F)$ that has a neighbour in a different component of $F$ a $k$-local skip-and-glue fusion of $F$ centred around $u$.

Note that the image of a pair $(F, u)$ of a $k$-local skip-and-glue strategy is not necessarily a graph all whose components contain at least $k$ vertices.

Lemma 7. Let $G$ be a connected graph containing a cycle. If there is a $k$-local skip-andglue strategy for $G$, then $G$ is 2-connected.

Proof. Let $C$ be a cycle in $G$ and suppose that some vertex $v$ separates $G$. Let $P$ be a path with one end vertex in $C$ and one end vertex in a component of $G-v$ not containing $C$. Let $C^{\prime}$ be a cycle containing $C$ and a maximal number of vertices from $P$. As there is a $k$ local skip-and-glue strategy for $G$, all vertices of $P$ are contained in $C^{\prime}$. This contradicts that $v$ is a separating vertex as it does not separate $C^{\prime}$ but $P$ meets two components of $G-v$.

Lemma 8. Let $G$ be a locally finite 2 -connected graph. Then every neighbourhood of every end contains a cycle of length at least $n$ for all $n \in \mathbb{N}$.

Proof. For an end $\omega$ of $G$ and a neighbourhood $U$ of $\omega$ and some $n \in \mathbb{N}$, let $R_{1}, R_{2}$ be two vertex disjoint rays in $\omega$ that lie in $U$, which exist as $G$ is 2-connected [16]. Let $P_{1}, P_{2}, \ldots$ be an infinite sequence of vertex disjoint finite $R_{1}-R_{2}$ paths. Such a sequence exists, as in every neighbourhood of $\omega$ there is one such path, and for any collection of finitely many paths there is a neighbourhood of $\omega$ avoiding those. Let $P_{i}$ and $P_{j}$ be two of these path whose end vertices on $R_{1}$ have distance at least $n$. The unique cycle in $R_{1} \cup P_{j} \cup R_{2} \cup P_{i}$ has length at least $n$.

We will see later that a locally finite graph which satisfies the conditions of Theorem 1 always admits a 5-local skip-and-glue strategy, hence the following theorem can be used to extend the theorem to locally finite graphs. 
Theorem 9. Let $k \in \mathbb{N}$ and let $G$ be a locally finite connected graph.

(i) If $G$ has a k-local skip-and-glue strategy and contains a cycle of length at least $k$, then $G$ is hamiltonian.

(ii) If $G$ contains a $v-w$ path $P$ whose end vertices have depth at least $k+1$ in $P$ and $G+v w$ has a $k$-local skip-and-glue strategy with respect to $V(P)$, then $G$ contains a $v-w$ arc that is hamiltonian.

Proof. Note that, if $G$ is finite, the existence of $k$-local skip-and-glue strategy directly implies (i) and (ii). Thus, we may assume that $G$ is infinite.

We want to apply Proposition 6 . Therefore, we shall define a sequence $\left(C_{i}\right)_{i \in \mathbb{N}}$ of finite cycles such that

(I) $V\left(C_{i+1}\right)$ contains $V\left(C_{i}\right)$,

(II) an edge with depth at least $2 k$ in $C_{i}$ is contained in $C_{i+1}$ if and only if it is contained in $C_{i}$,

(III) every vertex of $G$ is contained in some $C_{i}$,

(IV) for every end $\omega$ of $G$ and every finite set $V^{\prime} \subseteq V$ there is a finite cut $F$ separating $\omega$ from $V^{\prime}$ and an index $i_{0}$ such that $\left|E\left(C_{i_{0}}\right) \cap F\right|=2$ and $E\left(C_{i_{0}}\right) \cap F=E\left(C_{i}\right) \cap F$ for every $i>i_{0}$.

We define the $C_{i}$ recursively. Besides this sequence, we shall define a second sequence $\left(\Lambda_{i}\right)_{i \in \mathbb{N}}$ of labellings $\Lambda_{i}: V \rightarrow \mathbb{N}$. For $i, q \in \mathbb{N}$ let

$$
X_{q}^{i}:=\left\{v \in V \mid \Lambda_{i}(v)=q\right\} .
$$

Then the sequences will satisfy the following properties for every $1 \leqslant q$ :

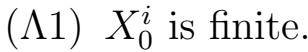

$(\Lambda 2) C_{i}\left[X_{q}^{i}\right]$ is a subpath of $C_{i}$.

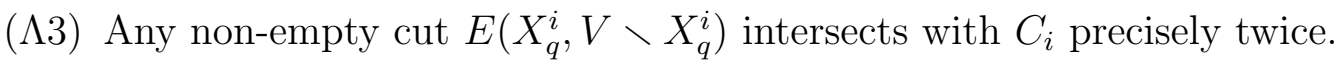

$(\Lambda 4)$ Every vertex in $X_{q}^{i}$ not in $C_{i}$ has distance more than $2 k$ from $V \backslash X_{q}^{i}$.

$(\Lambda 5)$ Every vertex of $C_{i}$ of depth $2 k$ or more in $C_{i}$ lies in $X_{0}^{i}$.

Let $C_{0}$ be any finite cycle of length at least $k$, which exists by assumption. For $j \in \mathbb{N}$ assume that all $C_{i}$ and $\Lambda_{i}$ with $i \leqslant j$ have been defined and that they satisfy (I), (II), and $(\Lambda 2)$ to $(\Lambda 5)$. Let $B(i) \subseteq G$ for $i \in \mathbb{N}$ be the restriction of $G$ to the vertices with distance at most $i$ from $C_{j}$. Let $\mathcal{D}=\left\{D_{1}, \ldots, D_{n}\right\}$ be the set of infinite components of $G \backslash B(2 k)$. Note that $\mathcal{D}$ is finite since $G$ is locally finite. Let $\mathcal{D}_{f}$ be the set of finite components of $G \backslash B(2 k)$. Due to Lemma 7, we know that $G$ is 2 -connected. So according 


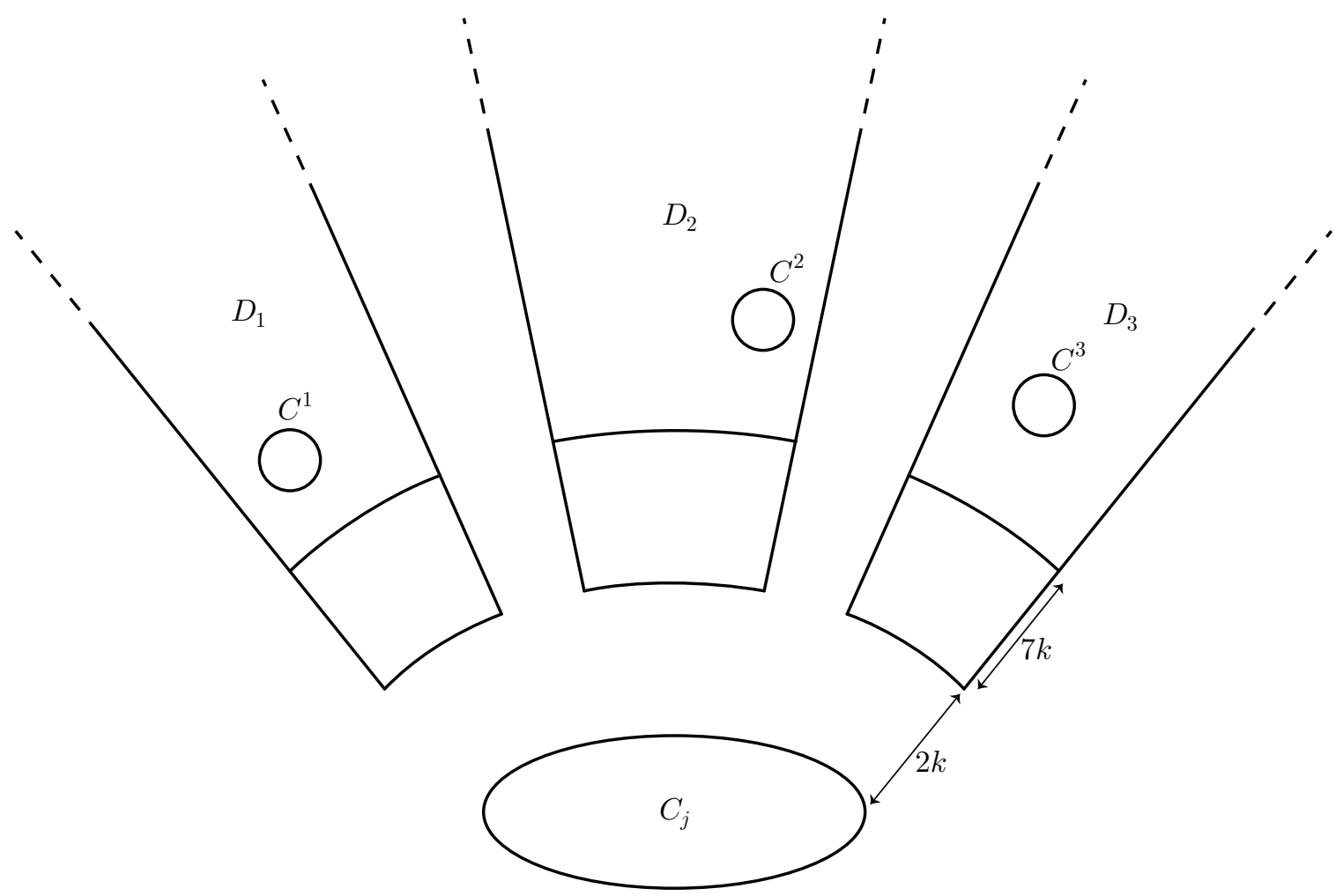

Figure 1: The cycle $C_{j}$ with the cycles $C^{i}$.

to Lemma 8, we find a cycle within every component $D_{\ell} \in \mathcal{D}$ of length at least $k$ that has distance at least $2 k$ to the boundary of $D_{\ell}$, adding successively all vertices with distance at most $k$ to this initial cycle via $k$-local skip-and-glue extensions we have a cycle $C^{\ell}$ in $D_{\ell}$ with at least $k$ vertices of depth at least $k$ in $C^{\ell}$, as shown in Figure 1.

Let $\mathcal{C}=\left\{C^{1}, \ldots, C^{n}\right\}$. Let $x_{1}, \ldots, x_{m}$ be an enumeration of some vertices of $\bigcup \mathcal{D}$ with

$$
(B(7 k) \cap \bigcup \mathcal{D}) \cup \bigcup \mathcal{C} \subseteq G\left[x_{1}, \ldots, x_{m}\right]
$$

such that for every $i \leqslant m$ each component of $G\left[x_{1}, \ldots, x_{i}\right]$ has a vertex in some $C^{\ell}$. By our $k$-local skip-and-glue strategy, there is a sequence

$$
C_{j} \cup \bigcup \mathcal{C}=F_{0}, \ldots, F_{m}=F
$$

of finite 2-regular graphs such that $F_{i+1}=F_{i}$ if $x_{i+1} \in V\left(F_{i}\right)$, and such that $F_{i+1}$ is obtained from $F_{i}$ by a $k$-local skip-and-glue extension via $x_{i+1}$ if $x_{i+1} \notin V\left(F_{i}\right)$. Note that all these extensions have length at most $k$ and thus $C_{j} \subseteq F$. Clearly, $F$ has at most $n+1$ components as $F_{0}$ has precisely $n+1$. Furthermore, the choice of the vertices $x_{i}$ gives us that the vertices of $F$ in $D_{i}$ induce a connected graph in $G$ for each $i \leqslant n$. Figure 2 shows the subgraph $F$.

We proceed with a finite sequence $F=F^{0}, \ldots, F^{p}=F^{\prime}$ of $k$-local skip-and-glue fusions: If some vertex $u$ of $F^{i} \cap \bigcup \mathcal{D} \subseteq G-B(2 k)$ has a neighbour in a different 


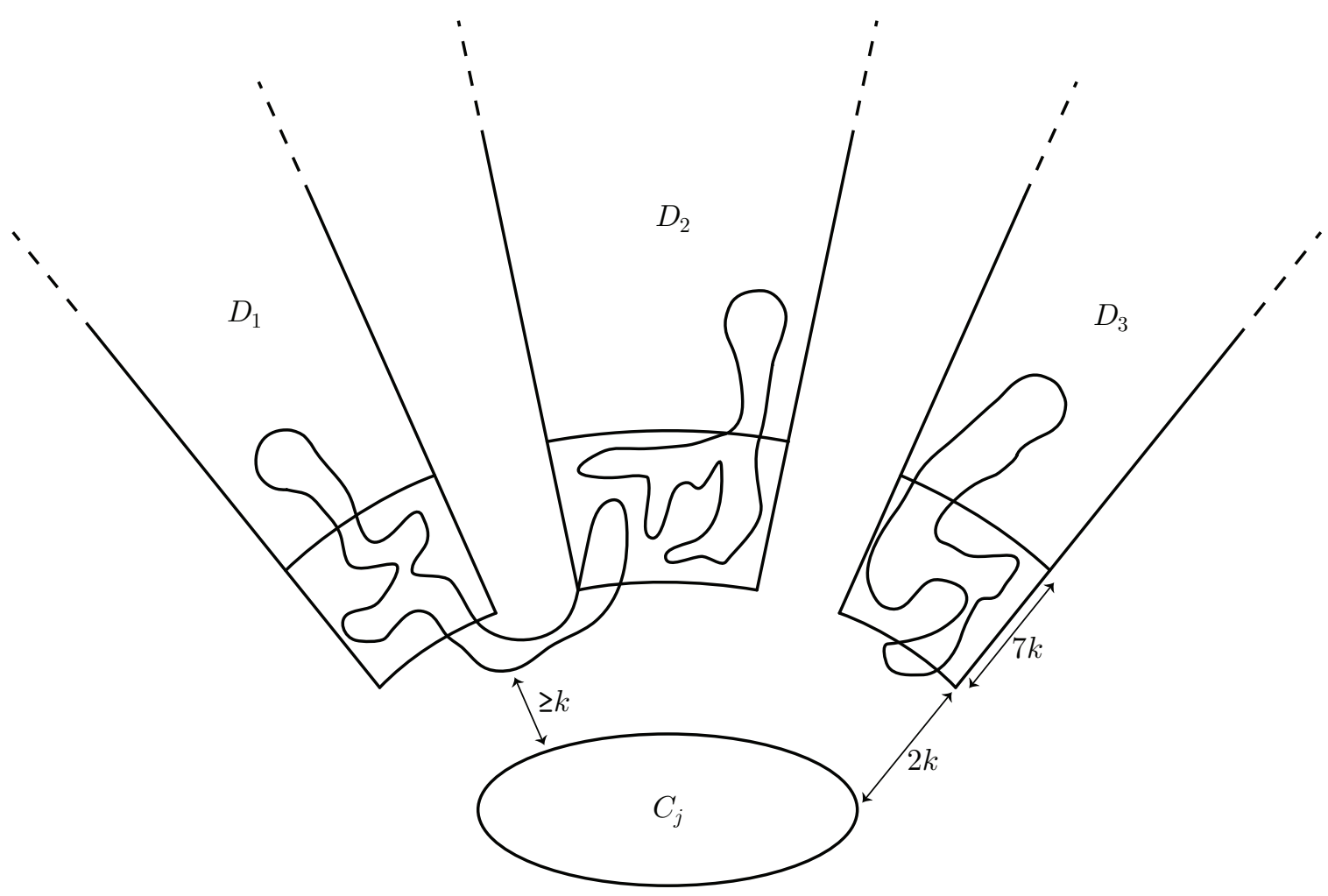

Figure 2: The subgraph $F$.

component of $F^{i}$, let $F^{i+1}$ be a $k$-local skip-and-glue fusion of $F^{i}$ centred around $u$. Since the number of components of $F$ is at most $n+1$ and reduces by at least 1 with each skip-and-glue fusion we have $p \leqslant n$. Then we have the following properties:

(1) Every $D_{i} \in \mathcal{D}$ contains vertices from exactly one component of $F^{\prime}$ and all vertices within $B(7 k) \cap D_{i}$ lie in this component.

(2) $C_{j}=F^{\prime} \cap B(k)$

(3) $F^{\prime}$ has at most $n+1$ components.

The subgraph $F^{\prime}$ is shown in Figure 3.

Next, we construct a finite sequence of $k$-local skip-and-glue extensions via vertices in $B(7 k) \cup \bigcup \mathcal{D}_{f}$ such that its first element is $F^{\prime}$ and its last element $E$ contains every vertex of $B(7 k) \cup \cup \mathcal{D}_{f}$. As every extension has length at most $k$, we have the following properties:

(4) All vertices with depth at least $k$ in $C_{j}$ lie in a common component of $E$.

(5) Only one component of $E$ meets $D_{i} \backslash B(3 k)$ for every $i \leqslant n$.

(6) $V(E)$ is connected in $G$. 


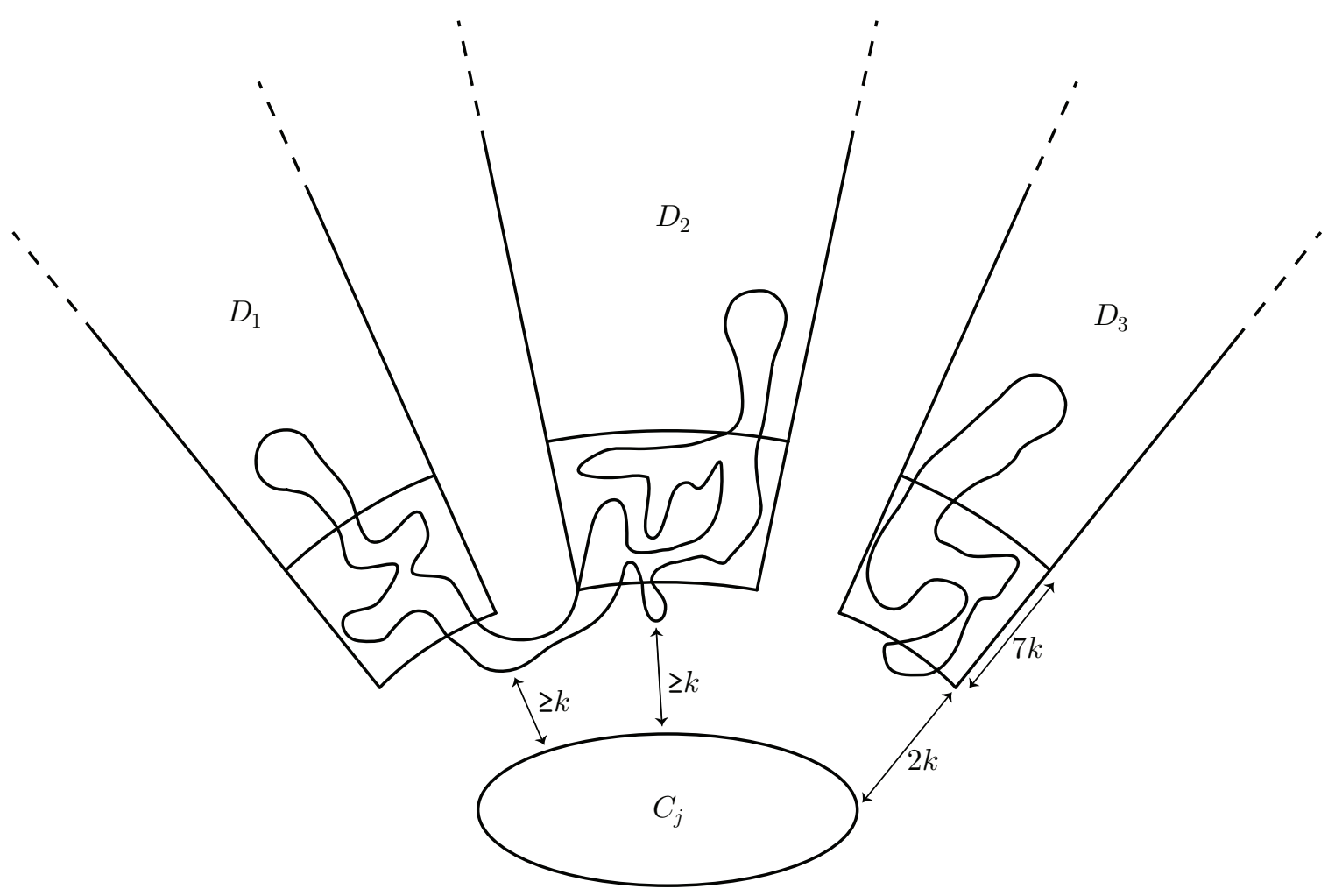

Figure 3: The subgraph $F^{\prime}$.

(7) $E$ has at most $n+1$ components.

Let $E=E_{0}, \ldots, E_{\ell}=C_{j+1}$ be a sequence of 2-regular subgraphs of $G$ such that $E_{\ell}$ is connected and $E_{i+1}$ is a $k$-local skip-and-glue fusion centred around a vertex that lies in the unique component $U_{i}$ of $E_{i}$ containing all vertices with depth at least $k$ in $C_{j}$ and that additionally lies in $B(4 k)$. It is possible to choose the centre in $B(4 k)$, as no $D_{i} \backslash B(4 k)$ contains vertices of more than one component of $E_{i}$ during this process since all skipand-glue fusions are $k$-local and by induction. Note that this is well-defined as $V\left(U_{i}\right)$ is contained in the fused cycle $U_{i+1}$. This finishes the construction of the cycles. The properties (I), (II), and (III) are direct consequences of the construction of $C_{j+1}$ because of $V(B(7 k)) \subseteq V\left(C_{j+1}\right)$.

To proof (IV) let us define a finite sequence $\Lambda^{0}, \ldots, \Lambda^{\ell}=\Lambda_{j+1}$ of labellings $\Lambda^{i}: V \rightarrow \mathbb{N}$ (with the same $\ell$ as above). For $i, q \in \mathbb{N}$ let

$$
Y_{q}^{i}:=\left\{v \in V \mid \Lambda^{i}(v)=q\right\} .
$$

We shall construct these labellings such that they satisfy the following conditions for $i \in \mathbb{N}, q>0$, and $1 \leqslant r \leqslant \ell$. Note that $\ell \leqslant n$.

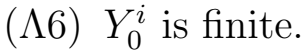

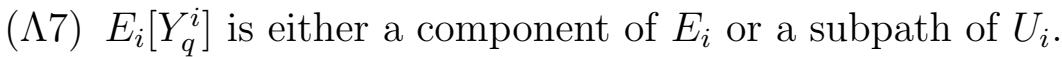




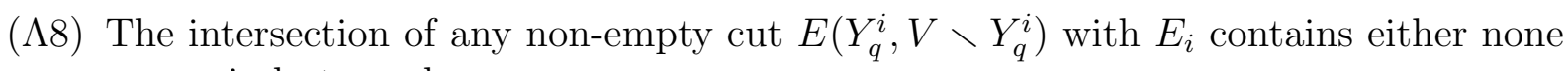
or precisely two edges.

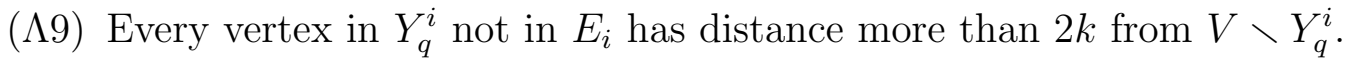

$(\Lambda 10)$ Every vertex of $C_{j}$ of depth $2 k$ or more in $C_{j}$ lies in $Y_{0}^{i}$.

Let $K_{0}, \ldots, K_{r}$ be the components of $E_{0}$ such that $K_{0}$ contains all vertices with depth at least $k$ in $C_{j}$, cp. (4). Let us define a labelling $\Lambda^{0}: V \rightarrow \mathbb{N}$. For a vertex $x \in V\left(K_{i}\right)$, let $\Lambda^{0}(x)=i$. For every component $D_{i} \in \mathcal{D}$, there is a unique label $\lambda_{0} \in \mathbb{N}$ with $\Lambda^{0}(x)=\lambda_{0}$ for all $x \in V\left(D_{i} \backslash B(3 k)\right) \cap V\left(K_{\lambda_{0}}\right)$, cp. (5). Let $\Lambda^{0}$ map every $y \in V\left(D_{i} \backslash E_{0}\right)$ to $\lambda_{0}$. Clearly, $\Lambda^{0}$ satisfies $(\Lambda 6)$ to $(\Lambda 10)$.

Assume $\Lambda^{s}$ has been defined for $0 \leqslant s<\ell$. The cycle $U_{s+1}$ is the union of a reduction $R_{s}$ of $U_{s}$, a reduction $R$ of some other component $U$ of $E_{s}$ and two disjoint paths $P, Q$ minus two edges $e_{s} \in E\left(R_{s}\right)$ and $e \in E(R)$. If both end vertices of $e_{s}$ have the $\Lambda^{s}$-label 0 , let $\lambda$ be the unique label of the vertices of $U$, cp. $(\Lambda 7)$. Otherwise, let $\lambda$ be the largest $\Lambda^{s}$-label at an end vertex of $e_{s}$. Let $W$ be the set consisting of the vertices on $P, Q$, and $R$ as well as all the vertices labelled with the label of $U$. Let $\Lambda^{s+1}$ equal $\Lambda^{s}$ outside of $W$ and let $\Lambda^{s+1}$ map every vertex of $W$ to $\lambda$.

Clearly, properties $(\Lambda 6)$ and $(\Lambda 7)$ are kept throughout the construction. Moreover, $(\Lambda 8)$ is a direct consequence of $(\Lambda 7)$. Since all fusions are $k$-local and centred around vertices in $B(4 k)$ we have $(\Lambda 9)$. Every vertex of depth at least $k$ in $C_{j}$ lies in all $U_{i}$ and thus the fusions are centred around vertices with depth at most $k$ in $C_{j}$ as one of the paths used for the skip-and-glue fusion has a vertex outside of $U_{i}$. Since every vertex with depth at least $2 k$ in $C_{j}$ lies on the reduction of $U_{i}$ for all $k$-local fusions of $E_{i}$, we have $(\Lambda 10)$. Thus we have $(\Lambda 1)$ to $(\Lambda 5)$ as $E_{\ell}=C_{j+1}$ is connected, $\Lambda^{\ell}=\Lambda_{j+1}$, and $\ell \leqslant n$.

Now we are able to prove (IV). Let us consider any finite set $S \subseteq V$ and any end $\omega$ of $G$. By (I) and (III) and as $G$ is locally finite, we find an index $j$ such that every vertex of $S$ has depth more than $2 k$ in $C_{j}$. So by $(\Lambda 5)$, it lies in $X_{0}^{j}$. The end $\omega$ lies in some infinite component $K$ of $G-B(7 k)$. By $(\Lambda 4)$, all vertices of $K$ have the same $\Lambda_{j}$-label $q$. As $\mathcal{B}=E\left(X_{q}^{j}, V \backslash X_{q}^{j}\right)$ is non-empty, it intersects with $C_{j}$ precisely twice due to $(\Lambda 3)$. By $(\Lambda 4)$, the vertices incident with $\mathcal{B}$ have depth $2 k$ or more in $C_{j}$. So (II) implies that $\mathcal{B}$ meets $C_{i}$ in precisely these two edges for every $i \geqslant j$. This shows (IV) and completes the proof of (i).

To prove (ii) we pick a sequence $P+v w=F_{0}, \ldots, F_{n}$ of cycles such that $F_{i+1}$ is a $k$ local skip-and-glue extension of $F_{i}$ and $F_{n}$ covers every vertex of distance at most $2 k+1$ from $v w$. Clearly, $v w$ is an edge of $F_{n}$. Following the proof of (i) there is a sequence $F_{n}=C_{0}, C_{1}, \ldots$ of finite cycles satisfying (I) to (IV). Thus for their limit $C$ its closure $\bar{C}$ is a Hamilton circle and $C$ contains $v w$ by (II). This completes the proof of (ii) as $C-v w$ is a Hamilton arc of $G$ with end vertices $v$ and $w$. 


\section{Locally connected graphs}

For a subgraph $F$ of a graph $G$, let us call a vertex $x \in V(F)$ skippable if $x$ has degree 2 in $F$ and its two neighbours $y, z$ are adjacent in $G$ but not in $F$. We call $y z$ an $x$-bypass. Note that, if $F$ is 2-regular, $x$ is skippable if and only if $y x z$ is a skippable path in $F$.

Lemma 10. Every connected locally connected locally finite claw-free graph $G$ has a 5-local skip-and-glue strategy.

Furthermore, for $x, y \in V(G)$ the graph $G+x y$ has a 5-local skip-and-glue strategy with respect to $V(P)$ for some $x-y$ path $P$ that contains all vertices of distance at most 3 from $x$ or $y$.

Proof. Let $G$ be a connected locally connected locally finite claw-free graph on at least three vertices. Let $F$ be a finite subgraph of $G$ with maximum degree 2 all whose components contain at least four vertices. Let $C$ be any component of $F$. Note that $C$ is either a cycle or a path. If $C$ is a path we require its end vertices to have depth at least 3 in $C$ and add the edge between those end vertices to $G$. Note that in the later construction, none of these new edges appears as they are too deep in their respective component. For the readability of the proof we omit their presence from now on and consider $G$ to be the graph with these edges added and $F$ to be 2-regular. With a slight stretch of terminology, we consider $G$ to be claw-free, although it is $G$ without these additional edges that is claw-free.

On each component $C$ we choose one order $\leqslant_{C}$ of the two available canonical cyclic orders. For every vertex $v \in V(C)$ denote by $v^{-}$its predecessor with respect to $\leqslant_{C}$ and by $v^{+}$its successor.

Let $u \notin V(F)$ be a vertex of $G$ that has a neighbour $v$ on $F$. To show that there is a $k$-local skip-and-glue strategy we have to provide a $k$-local skip-and-glue extension of $F$ via $u$. As $G$ is locally connected $N(v)$ is connected; it contains $u, v^{-}$, and $v^{+}$. Thus there is a shortest $u-\left\{v^{-}, v^{+}\right\}$path $P=p_{0} p_{1} \ldots p_{k}$ with all its vertices in $N(v)$. We may assume that $p_{0}=u$ and $p_{k}=v^{+}$by choosing the other canonical order for the component containing $u$ if necessary. Clearly, $P$ does not contain $v^{-}$. By minimality $P$ is induced and thus if $k \geqslant 4$, we have the claw $G\left[v, p_{0}, p_{2}, p_{4}\right]$ in $G$. Hence $k \leqslant 3$ and the length of $P$ is at most 3 .

It remains to construct a $k$-local skip-and-glue extension. We consider two cases: either all inner vertices of $P$ are skippable and no two consecutive vertices of $P$ are adjacent on $F$ or this is not the case. The two possible extensions in these cases are depicted in Figure 4.

In the first case, we can replace every skippable path $p_{i}^{-} p_{i} p_{i}^{+}$by the edge $p_{i}^{-} p_{i}^{+}$. Furthermore, we replace the edge $v v^{+}$by the path $v u P v^{+}$to obtain a 4-local skip-and-glue extension of $F$ via $u$. This covers the case that $u$ is adjacent to $v^{-}$or $v^{+}$.

Thus we may assume that $P$ has an inner vertex and since $G$ is claw-free it holds that $v^{-} v^{+} \in E(G)$. Since the component of $F$ containing $v$ has at least four vertices, $v$ is skippable. If some inner vertex $p_{i}$ of $P$ lies on $F$ and is not skippable, then the path $p_{i}^{-} p_{i} p_{i}^{+}$is not skippable. Thus, we have $p_{i}^{-} p_{i}^{+} \notin E$. 
It remains to construct a $k$-local skip-and-glue extension if either some vertex of $P$ is not skippable or some edge of $P$ lies on $F$. Let $p_{i}$ be the first vertex on $P$ that is either not skippable or incident with an edge of $P \cap F$. As $G\left[v, p_{i}, p_{i}^{-}, p_{i}^{+}\right]$is not a claw, there is an edge $v p_{i}^{-}$or $v p_{i}^{+}$in the first case. As $P$ lies in the neighbourhood of $v$, there is an edge $v p_{i}^{-}$or $v p_{i}^{+}$in the second case, too. As $v$ is skippable, we reduce $F$ by replacing $v^{-} v v^{+}$ by the edge $v^{-} v^{+}$and $p_{j}^{-} p_{j} p_{j}^{+}$by the edge $p_{j}^{-} p_{j}^{+}$for all $j<i$ where $p_{j}$ is skippable. If $v$ and $p_{i}^{-}$are adjacent, then we replace the edge $p_{i} p_{i}^{-}$by the path $p_{i}^{-} v u P p_{i}$. Otherwise, $v$ and $p_{i}^{+}$are adjacent and we replace the edge $p_{i} p_{i}^{+}$by the path $p_{i} P u v p_{i}^{+}$. Note that $i \leqslant 2$. Thus, we obtain a 4-local skip-and-glue extension of $F$ via $u$ in both cases. Both cases with the resulting extensions, which are coloured in red, are shown in Figure 4.
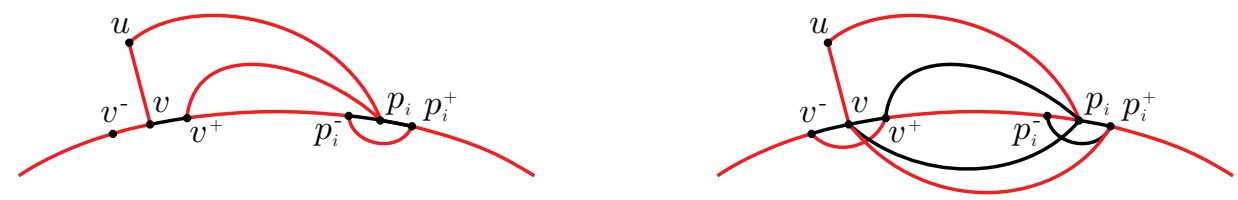

Figure 4: Two special cases for skip-and-glue extensions.

It remains to show that 5-local skip-and-glue fusions of $F$ exist. Therefore, let us assume that $F$ has more than one component each containing at least four vertices. Let $v \in V(F)$ be a vertex in a component $K$ of $F$ with a neighbour in some other component of $F$. Following the above argument for every vertex in $N(v) \cap V(F \backslash K)$ we have a 4-local skip-and-glue extension $K^{\prime}$ of $K$ by $P$ via some vertex $u \in N(v) \cap V(F \backslash K)$ over some edge $e \in E(K)$ such that the inner vertices of $P$ lie in $N(v) \cup\{v\}$ and $u$ is the only vertex in $P$ from $F \backslash K$. Indeed, we get a path $P$ completely contained in $N(v) \cup\{v\}$, and such a path of minimal length contains only one vertex from $F \backslash K$. We forget that its end vertices are contained in $N(v) \cup\{v\}$ for later convenience.

Similarly, there is a path $Q$ in the neighbourhood of $u$ from $\left\{u^{-}, u^{+}\right\}$to one of the two neighbours of $u$ on $K^{\prime}$, which are its neighbours on $P$.

For the following construction let us choose such $P$ and $Q$ with some minimality conditions:

(i) Let $P$ be shortest possible.

(ii) With respect to (i) let $Q$ be shortest possible.

Clearly, $|E(P)|=2$ if and only if there is an edge $e$ in $K$ with both its end vertices adjacent to $u$. Note that no inner vertex of $Q$ lies on $P$, as this vertex must be one of the neighbours of $u$ on $P$, which would contradict the minimality of $Q$.

Suppose that there is an inner vertex $q$ of $Q$ in $K^{\prime}$ that is either not skippable or incident with an edge of $Q$ that lies in $K^{\prime}$. As seen above $q \in V(K) \backslash V(P)$. Let $a, b$ be the two neighbours of $q$ on $K^{\prime}$. As $G[u, q, a, b]$ is not a claw, there is an edge $u a$ or $u b$ if $q$ is not skippable. As $Q$ lies in the neighbourhood of $u$, there is an edge $u a$ or $u b$ if $q$ is incident with an edge of $Q$ on $K^{\prime}$, too. By symmetry we may assume that $a$ is 
adjacent to $u$. If $q a \in E(K)$ we have a contradiction to the minimality of either $P$ or $Q$ as we could have chosen $P=q u a$ and shortened $Q$ to end in $q$. Thus we may assume that $q a$ is a $z$-bypass for some $z \in V(P)$. This contradicts the minimality of $P$ as $q u P z$ is shorter since both $u$ and $z$ are inner vertices of $P$. (Note that in both cases $q u a$ and $q u P z$, respectively, have the property that all their inner vertices lie in the neighbourhood of $v$.)

Thus, every inner vertex of $Q$ in $K^{\prime}$ is skippable and no edge of $Q$ is an edge of $K^{\prime}$.

We distinguish two cases: either all inner vertices of $Q$ are skippable in $F$ and in $K^{\prime}$ and no edge of $Q$ lies in $F \backslash K$ or $K^{\prime}$ or this is not the case. For both cases the 5-local skip-and-glue fusions are shown in Figure 5.

In the first case, let $F^{\prime}$ be the reduction of $F$ where the inner vertices of $Q$ and $P$ in $F$, except for $u$, are replaced by their bypasses and let $L$ be the set consisting of the edge $e$ and the edges from $u$ to the end vertices of $Q$. Then $\left(F^{\prime} \cup P \cup Q\right)-L$ is a skip-and-glue fusion of $F$. It is 5-local as $Q$ has length at most 3 and every component of $P-L$ has length at most 3. Clearly, it is centred around $v$.

Thus we may assume that there is an inner vertex on $Q$ in $F \backslash K$ that is either not skippable or incident with an edge of $Q \cap(F \backslash K)$. First note that every vertex of $Q$ except for its first vertex (either $u^{-}$or $u^{+}$) is adjacent to a neighbour of $u$ in $F$ if $u$ is not skippable in $F$, namely either $u^{+}$or $u^{-}$. So if $u$ is not skippable, then the end vertex of $Q$ on $K^{\prime}$ is adjacent to either $u^{+}$or $u^{-}$and thus $Q$ had no inner vertex by its minimality. But as $Q$ contains an inner vertex, $u$ must be skippable in $F$.
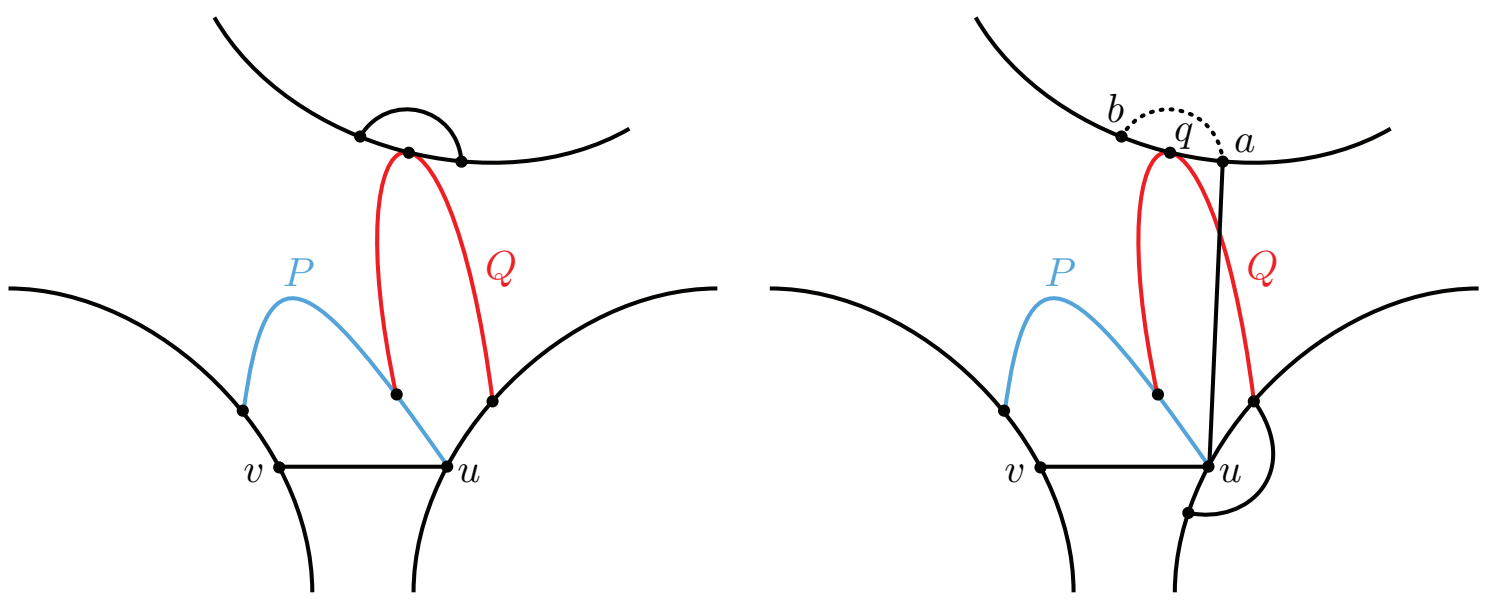

Figure 5: Two special cases for skip-and-glue fusions.

Let $q$ be the last inner vertex of $Q$ in $F \backslash K$ that is either not skippable or incident with an edge of $Q \cap(F \backslash K)$. Thus its subpath $q Q p$ with $p \in V(P)$ does not contain any such vertices. Let $a, b$ be the two neighbours of $q$ on $F$. As $G[u, q, a, b]$ is not a claw, there is an edge $u a$ or $u b$ if $q$ is not skippable. As $Q$ lies in the neighbourhood of $u$, there is an edge $u a$ or $u b$ if $q$ is incident with an edge of $Q$ on $F$, too. By symmetry we may assume that $a$ is adjacent to $u$. This situation is shown in Figure 5 on the right.

Let $F^{\prime}$ be the reduction of $F$ where the inner vertices of $Q$ and $P$ in $F$ except for $u$ are replaced by their bypasses and set $L:=\{e, u p, a q\}$. Then $\left(F^{\prime} \cup P \cup q Q p\right)-L+a u$ is a 
skip-and-glue fusion of $F$. It is 4-local as $q Q p$ has length at most 2 and the component of $P-u p$ containing $p$ has length at most 2 and the other component of $P-u p$ has length at most 3. Clearly, it is centred around $v$.

The following is a corollary extracted from the previous proof.

Corollary 11. Every connected locally connected locally finite claw-free graph on at least $k \geqslant 3$ vertices contains a cycle of length at least $k$.

Now we can combine our previous results to prove our first main theorem.

Theorem 12. Every connected locally connected locally finite claw-free graph on at least three vertices has a Hamilton circle.

Proof. Let $G$ be a connected locally connected locally finite claw-free graph on at least three vertices. By Corollary 11 the graph $G$ contains a cycle of length at least $\min \{|G|, 5\}$. Due to Lemma 10, there is a 5-local skip-and-glue strategy for $G$. Thus, the assertion is a direct consequence of Theorem 9 .

Let us now turn our attention to Hamilton arcs. We deduce from the proof of Theorem 2 in [1] the following proposition that is valid for all locally finite graphs.

Proposition 13. Let $G$ be a 3-connected locally connected locally finite claw-free graph on at least four vertices. For every two $x, y \in V(G)$ there is an $x-y$ path of length at least 3 that contains $N(x) \cup N(y)$.

In the proof that the result of Proposition 13 implies that the graph has a Hamilton arc, Asratian [1, Theorem 2.5] showed that, given the conclusion of Proposition 13 in a finite locally connected claw-free graph, it has a Hamilton path between $x$ and $y$. In our situation, the proof of [1, Theorem 2.5] together with Proposition 13 yields the following:

Proposition 14. Let $G$ be a 3-connected locally connected locally finite claw-free graph on at least four vertices. For every two $x, y \in V(G)$ there is an $x-y$ path of length at least 3 that contains all vertices of distance at most 2 to either $x$ or $y$.

Using our terminology, Proposition 14 implies the existence of some $x-y$ path $P$ such that the depth in $P$ of its end vertices is at least 3 . This enables us to prove our second main theorem.

Theorem 15. A locally connected locally finite claw-free graph on at least four vertices is Hamilton-connected if and only if it is 3-connected.

Proof. Obviously, no Hamilton-connected graph has connectivity at most 2 as no Hamilton arc can connected any two vertices whose removal disconnects the graph.

For the other direction, let $G$ be a connected locally connected locally finite claw-free graph and let $x, y \in V(G)$ be distinct vertices. As mentioned before, Proposition 14 implies that we find an $x-y$ path $P$ such that $x$ and $y$ have depth at least 3 in $P$. Since $G+x y$ has a 5-local skip-and-glue strategy with respect to $V(P)$ by Lemma 10 there is a sequence $P+x y=C_{0}, \ldots, C_{n}$ of cycles in $G+x y$ such that $C_{i+1}$ is a 5 -local skip-and-glue extension of $C_{i}$ and such that $C_{n}$ contains all vertices of distance at most 6 from $x$ or $y$. Thus the assertion follows from Theorem 9. 


\section{Further sufficient conditions for the existence of a Hamilton circle}

In this section, we deduce some corollaries from the main theorems of Section 4 . To shorten this section, we say that a graph $G$ satisfies $(\star)$ if the following statements are true:

(i) $G$ has a Hamilton circle.

(ii) For every two vertices $u, v \in V(G)$ that do not separate $G$, there is a Hamilton $u-v$ arc in $|G|$.

(iii) $G$ is Hamilton-connected and $|V(G)| \geqslant 4$ if and only if it is 3-connected.

It is well-known that line graphs are claw-free. Thus, we directly obtain the following corollary (whose finite version for Hamilton cycles is due to Oberly and Sumner [22, Corollary 1]):

Corollary 16. Let $G$ be a locally finite connected locally connected line graph on at least three vertices. Then $G$ satisfies $(\star)$.

The proof that the assumptions of the following corollary imply that $L(G)$ is locally connected is the same as for finite graphs. Thus, we obtain the following corollary (whose finite version for Hamilton cycles is due to Oberly and Sumner [22, Corollaries 2 and 3]):

Corollary 17. Let $G$ be a locally finite connected graph on at least three vertices such that either every edge lies on a triangle or $G$ is locally connected. Then $L(G)$ satisfies $(\star)$.

For the following two corollaries the proofs that their assumptions imply that $L(L(G))$, $L\left(G^{2}\right)$, respectively, is locally connected is the same as for finite graphs, see [22, Corollaries 4 and 5]. The finite version for Hamilton cycles of Corollary 18 is due to Chartrand and Wall [6]) and that of Corollary 19 is due to Nebeský [21].

Corollary 18. Let $G$ be a locally finite connected graph with minimum degree at least 3 . Then $L(L(G))$ satisfies $(\star)$.

Note that for a graph $G$, its square $G^{2}$ has $V(G)$ as its set of vertices and two distinct vertices are adjacent in $G^{2}$ if their distance in $G$ is at most 2.

Corollary 19. Let $G$ be a locally finite connected graph on at least three vertices. Then $L\left(G^{2}\right)$ satisfies $(\star)$.

The last corollary of the results of Section 4 carries over the result by Matthews and Sumner [20, Corollary 1] for Hamilton cycles in finite graphs to locally finite graphs. Again, their proof carries over almost verbally.

Corollary 20. Let $G$ be a locally finite connected graph on at least three vertices. If $G^{2}$ is claw-free, then it satisfies $(\star)$. 


\section{References}

[1] A.S. Asratian. Every 3-connected, locally connected, claw-free graph is Hamiltonconnected. J. Graph Theory, 23:191-201, 1996.

[2] E. Berger and H. Bruhn. Eulerian edge sets in locally finite graphs. Combinatorica, $31: 21-38,2011$.

[3] R.C. Brewster and D. Funk On the Hamiltonicity of line graphs of locally finite, 6-edge-connected graphs. J. Graph Theory, 71:182-191, 2012.

[4] H. Bruhn and M. Stein. On end degrees and infinite circuits in locally finite graphs. Combinatorica, 27:269-291, 2007.

[5] H. Bruhn and X. Yu. Hamilton cycles in planar locally finite graphs. SIAM J. Discrete Math., 22:1381-1392, 2008.

[6] G. Chartrand and C.E. Wall. On the Hamiltonian index of a graph. Studia Sci. Math. Hungar., 8:43-48, 1973.

[7] Q. Cui, J. Wang, and X. Yu. Hamilton circles in infinite planar graphs. J. Combin. Theory Ser. B, 99:110-138, 2009.

[8] R. Diestel. Graph Theory. Springer, 4th edition, 2010.

[9] R. Diestel. Locally finite graphs with ends: a topological approach. I, II and III. Discrete Math 311: 1423?1447; 310: 2750-2765; 312: 21-29 (2010?2012), arXiv:0912.4213.

[10] R. Diestel and D. Kühn. Graph-theoretical versus topological ends of graphs. J. Combin. Theory Ser. B, 87:197-206, 2003.

[11] R. Diestel and D. Kühn. On infinite cycles I. Combinatorica, 24:68-89, 2004.

[12] R. Diestel and D. Kühn. On infinite cycles II. Combinatorica, 24:91-116, 2004.

[13] R. Diestel and D. Kühn. Topological paths, cycles and spanning trees in infinite graphs. European J. Combin., 25:835-862, 2004.

[14] H. Freudenthal. Über die Enden topologischer Räume und Gruppen. Math. Z., 33:692-713, 1931.

[15] A. Georgakopoulos. Infinite hamilton cycles in squares of locally finite graphs. Adv. Math., 220:670-705, 2009.

[16] R. Halin. A note on Menger's theorem for infinite locally finite graphs. Abh. Math. Sem. Univ. Hambg., 40:111-114, 1974.

[17] K. Heuer. A sufficient condition for Hamiltonicity in locally finite graphs. European J. Combin. 45:97-114, 2015.

[18] K. Heuer. A sufficient local degree condition for the hamiltonicity of locally finite claw-free graphs. European J. Combin. (to appear).

[19] F. Lehner. On spanning tree packings of highly edge connected graphs. J. Combin. Theory Ser. B) 105:93-126, 2014. 
[20] M.M. Matthews and D.P. Sumner. Hamiltonian results in $K_{1,3}$-free graphs. J. Graph Theory, 8:139-146, 1984.

[21] L. Nebeský. On the line graph of the square and the square of the line graph of a connected graph. Časopis Pěst. Mat., 98:285-287, 1973.

[22] D.J. Oberly and D.P. Sumner. Every connected, locally connected nontrivial graph with no induced claw is Hamiltonian. J. Graph Theory, 3:351-356, 1979.

[23] M. Stein. Extremal infinite graph theory. Discrete Math., 311:1472-1496, 2011.

[24] C. Thomassen. Hamiltonian paths in squares of infinite locally finite blocks. Ann. Discrete Math., 3:269-277, 1978. 